\title{
Exploiting the Bandwidth-Distance Relationship in Underwater Acoustic Networks
}

\author{
Paolo Casari $^{\ddagger}$, Milica Stojanovic ${ }^{\star}$ and Michele Zorzi ${ }^{\ddagger}$ \\ ${ }^{\ddagger}$ Department of Information Engineering — University of Padova — Via Gradenigo 6/B, I-35131 Padova, Italy \\ ${ }^{\star}$ Sea Grant College Program — Massachusetts Institute of Technology — 292 Main St. E38-300, Cambridge, MA, 02139-4309 \\ E-mail: $\{$ casarip, zorzi\}@dei.unipd.it, millitsa@mit.edu
}

\begin{abstract}
In this paper we study the relationship between effective use of the available bandwidth, energy consumption, and transmission delay in a Underwater Wireless Acoustic Sensor Network (UWASN). We compare different solutions to transport data to a sink node, namely multihop transmissions through multiple relays and the use of direct relay-to-sink links that require more power but reach the sink in one hop. We also address the effects of different error control policies. Our analysis shows that the energy consumption can be traded off for delay by choosing different policies and by varying some key parameters in each policy.
\end{abstract}

Index Terms-Acoustic telemetry and communication; Access, custody, and retrieval of data; Information management.

\section{INTRODUCTION AND RELATED WORK}

W ATER column sampling and underwater environmental monitoring pose difficult challenges for oceanographers and marine biologists. However, recent developments in acoustic modem technology are paving the way to the deployment of the first underwater sensor networks, opening new perspectives to the way monitoring tasks can be carried out. Underwater networks would operate unattended according to the ad hoc paradigm well known in radio networks, but those networks call for the design of communication schemes and networking protocols that meet the challenges of the underwater channel and application performance requirements. Such needs may vary depending on the operations attended, from real-time data delivery by detection and tracking systems, to energy-efficient data harvesting as required by long term environmental sampling operations. Underwater Wireless Acoustic Sensor Networks (UWASNs) may also be augmented with autonomous underwater vehicles (AUVs); for example, this unmanned machinery could interrogate the sensors ondemand, or cooperate to haul data to control stations.

In order to design effective and energy-efficient underwater communication networks, the physics of acoustic propagation must be taken into account. Two factors influence the design of network architecture and network protocols: the low speed of sound $(1500 \mathrm{~m} / \mathrm{s})$, and the dependence of the available bandwidth on the transmission distance. The latter stems from the frequency-dependent nature of the acoustic path loss and of the ambient noise [1].

Most of the current research on underwater networks has focused on MAC design and multiple access in general [2][6] or on routing [7]-[9]. The bandwidth-distance relationship

This work was partially supported by NOAA's Sea Grant College Program, Project no. NA060AR4170019, and by NSF grants no. 0520075 and 0427502. of the acoustic channel and its impact on the design of an underwater network has been addressed in [10] and [11]. In [10] it is shown that in multihop UWASNs, there exists an optimal hop distance from an energy consumption point of view. The authors also infer that routing protocols should be designed to match such a distance, or if possible, to approach it from below (choosing closer neighbors), considering linear as well as 2- and 3-dimensional topologies. In [11] the use of two different bands is investigated to broadcast data along with redundancy packets for error protection. The data packets are relayed using a low-power, high-bandwidth link with a short reach, whereas the redundancy is conveyed to farther nodes using a high-power, low-frequency link. This broadcasting scheme is shown to yield advantages over simpler solutions based on ARQ or single-band hybrid ARQ/FEC schemes.

In this paper, we investigate the impact of the bandwidthdistance relationship on the design of a relay acoustic link from a broad point of view. The analysis we carry out is aimed at highlighting relevant tradeoffs emerging from the use of different data forwarding policies. We focus on a linear sensor network in which there are $r$ nodes distributed evenly over a distance $d_{\ell}$. The information collected by the sensors has to be sent to an end node which acts as a common sink. In this scenario, we analytically evaluate and compare a number of forwarding techniques that make different use of the bandwidth available at different distances, with the aim to find how delivery delay and energy consumption per packet vary with each policy, and how the parameters of each policy affect its performance.

\section{THE UnderWATER CHANNEL}

The power of an acoustic signal propagating underwater decays with distance according to the relation

$$
A(d, f)=d^{\kappa} a(f)^{d},
$$

where $d$ is the propagation distance, $\kappa$ models the geometry of propagation, and $a(f)$ is the absorption factor, approximated through Thorp's formula [12]. In this relation, the term $d^{k}$ can be seen as the counterpart of the attenuation incurred by radio waves in the air, whereas the term $a(f)^{d}$ is specific to acoustic waves in water, and models the attenuation as a function of the signal frequency, $f$.

The noise affecting the reception process has a frequencydependent power spectral density, resulting from the superposition of multiple environmental sources. Combined, these 
two features cause the bandwidth available for communication to decrease with distance. The signal-to-noise ratio (SNR) of an incoming acoustic tone of frequency $f$, transmitted from a distance $d$, can be expressed as

$$
\operatorname{SNR}(d, f)=\frac{P_{T} / A(d, f)}{N(f) \Delta f},
$$

where $P_{T}$ is the transmit power and $N(f)$ is the noise power spectral density (assumed constant in a narrow band $\Delta f$ around $f$ ). In (2), the factor $1 / A(d, f) N(f)$ is the frequencydependent component. For reference, see [1, Fig. 3], where this factor is plotted against frequency for different values of $d$, showing the existence of an optimal choice for the center frequency $f_{0}(d)$, and the bandwidth $B(d)$ around it. For example, the bandwidth can be defined according to the empirical $-3 \mathrm{~dB}$ rule, i.e., $B(d)=\left\{f: S N R(d, f)>S N R\left(d, f_{0}\right) / 2\right\}$. Both $f_{0}$ and $B$ are a function of the distance $d$. In particular, the optimal transmission bandwidth increases and is shifted toward higher frequencies of the acoustic spectrum as the sender and the receiver get closer, whereas it decreases and is moved toward the lower frequencies when the terminals are far apart. Let us assume that the signal occupies the optimal bandwidth $B(d)$, and that, for simplicity, its power spectral density $S(f)$ is constant, i.e., $S(f)=P_{T} / B(d)$. The SNR in this case is

$$
\operatorname{SNR}(d, B(d))=\frac{\frac{P}{B(d)} \int_{B(d)} A^{-1}(d, f) \mathrm{d} f}{\int_{B(d)} N(f) \mathrm{d} f} .
$$

Due to the one-to-one relationship between $d$ and $B(d)$, all integrals in (3) are determined when $d$ is fixed. In the following, we will use the shorthand notation

$$
A_{d}^{-1}=\int_{B(d)} A^{-1}(d, f) \mathrm{d} f, \quad N_{d}=\int_{B(d)} N(f) \mathrm{d} f .
$$

\section{OVERVIEW OF THE TRANSMISSION POLICIES}

As outlined in Section I, we focus on a line topology, with $r$ nodes, such that the distance between the farthest nodes is $d_{\ell}$. Each node generates $N$ packets that must be transmitted to the last node in the line, acting as the sink. To improve the overall system efficiency, the nodes are allowed to co-operate. They do so by relaying the information, so that a data packet from a node far away can travel to the sink over multiple hops, thus consuming less power, and having a greater bandwidth available. Relaying can be employed instead of, or in addition to, direct transmission. Specifically, we study the following transmission policies:

1) Relaying only: In this case, each node relays the packets from all the nodes upstream from it, in addition to transmitting its own $N$ packets. All nodes transmit at the same bit rate. An Automatic Repeat reQuest (ARQ) procedure is used to implement reliable transmission. Two forms of ARQ are considered: a simple Stop-andWait $(\mathrm{S} \& \mathrm{~W})$, and a group $\mathrm{S} \& \mathrm{~W}$, in which a selective acknowledgment (ACK) is sent for a group of $M$ packets [13]. The latter policy is hereon referred to as $M-\mathrm{S} \& \mathrm{~W}$.

2) Parallel transmission: In this case, each relay node has an option to forward some of its packets directly to the sink, while the rest are being sent to the next relay. Transmission over the two links is accomplished in two disjoint bands. The effect expected in this case is a reduction of the overall delay at the price of an increase in the energy consumption, as higher power is required to bridge longer links.

In the parallel transmission policy, the same bit rate is used on both links. However, the longer propagation delay on long links calls for more efficient error control strategies than $S \& W$ ARQ. ARQ is thus confined to short links where the delay can be tolerated. Instead, higher transmission power is used on long links. This power is set so that the probability that all packets are correctly received is $1-p_{t}$, for a target probability $p_{t}{ }^{1}$ On short links, the transmission power is set so as to achieve a target value $S N R_{0}$ for the Signal-to-Noise ratio, in order to ensure a sufficiently low probability of packet error.

\section{PERformance AnAlysis}

\section{A. Relaying with $S \& W A R Q$}

This simple policy is meant to serve as a baseline for reference and comparison. Let $p$ be the probability of success for a packet containing $L$ bits. According to (3), and assuming the use of a BPSK modulation with independent channel errors on the $L$ transmitted symbols, we have

$$
p=\left(1-\frac{1}{2} \operatorname{erfc} \sqrt{S N R_{0}}\right)^{L} \text {. }
$$

Note that (5) is the formula for the BPSK bit error rate assuming additive white Gaussian noise. Although the noise is non-white [1], when the signal bandwidth is sufficiently small, one can assume that the power spectral density of the noise is almost constant. In these conditions, expression (5) holds. The SNR is defined as that of an equivalent AWGN channel as in (3) [1].

Since the total distance is equally split between $h=r+1$ hops, where $r$ is the number of relays, each hop covers a distance of $d_{r}=d_{\ell} / h$. Hence, the transmit power is found as

$$
P_{T}\left(d_{r}\right)=\frac{S N R_{0} B\left(d_{r}\right) N_{d_{r}}}{A_{d_{r}}^{-1}}
$$

The average time required to transmit a packet between two successive nodes is $\left(T_{d}+2 \tau_{d_{r}}\right) / p+T_{a}$, where $\tau_{x}$ denotes the propagation time of an acoustic wave traveling a distance $x$, whereas $T_{d}$ and $T_{a}$ are the transmission time of a data and an ACK packet, respectively. Note that in the previous formula it is assumed that no ACK is transmitted if a packet is not received correctly. In this case, the transmitter sends the packet again after a time-out interval, that is long enough to accommodate the propagation delay between the sender and the relay downstream. Now, each node in the line must forward its own packets, and all those received from the previous nodes as well. Say that each node generates $N$ packets. Hence the number of packets to send is $N$ for the initial sender, $2 N$ for

\footnotetext{
${ }^{1}$ Note that in a more general setting that takes channel fading into account, sending all packets at higher power may not be the most efficient strategy. Some form of packet coding (e.g., Reed-Solomon [14]) would help instead. However, the evaluation of this scenario is out of the scope of the present paper and is left for future study.
} 


$$
\boldsymbol{P}_{M}=\left[\begin{array}{cccc}
1 & 0 & \cdots & \\
p & 1-p & 0 & \ldots \\
\vdots & \ddots & & \vdots \\
\left(\begin{array}{c}
M-1 \\
M-1
\end{array}\right) p^{M-1}(1-p)^{0} & \cdots & \left(\begin{array}{c}
M-1 \\
0
\end{array}\right) p^{0}(1-p)^{M-1} & 0 \\
\left(\begin{array}{c}
M \\
M
\end{array}\right) p^{M}(1-p)^{0} & & \cdots & \left(\begin{array}{c}
M \\
0
\end{array}\right) p^{0}(1-p)^{M}
\end{array}\right]
$$

the first relay, $3 N$ for the second relay, and so forth. Assuming that channel access is ideal, whereby each relay transmits its own packets right after the previous one has completed its transmission, the total delay $D^{(R)}$ is equal to the time required to transmit $N \cdot h(h+1) / 2$ packets:

$$
D^{(R)}=N \cdot \frac{h(h+1)}{2}\left(\frac{T_{d}+2 \tau_{d_{r}}}{p}+T_{a}\right) .
$$

The average energy consumed to have one packet correctly reach the following relay is found to be

$$
E^{(R)}=\frac{T_{d} P_{T}\left(d_{r}\right)+\left(T_{d}+2 \tau_{d_{r}}\right) P_{R}}{p}+\left(P_{T}\left(d_{r}\right)+P_{R}\right) T_{a},
$$

where the first term represents the average energy spent until the data packet correctly reaches the receiver, and the second one is the energy required to transmit the ACK packet. $P_{R}$ denotes the receive power. In the evaluation of both the total delay and the energy per packet, the return channel is assumed to be error-free, so that ACKs always reach their recipient correctly. Since ACKs are short and transmitted at the same power used for data, this assumption holds with high probability.

\section{B. Relaying with $M-S \& W$}

In this case, the ARQ policy is slightly different. The sender transmits a burst of $M$ packets back-to-back and waits for a reception confirmation. The recipient always replies with a selective ACK, to report which packets were correctly received and which were not. As opposed to plain $\mathrm{S} \& \mathrm{~W}$ $\mathrm{ARQ}$, the reply packet is needed even if no data packet is correctly received; otherwise the senders would not know which transmissions to repeat. In the following, we will call the sequential transmission of the data packet train and of the reply packet a "round".

To capture the average total delay and the average energy consumption per packet, we use the following semi-Markov model. Let $p_{i j}$ be the probability that, after a certain transmission round, $j$ packets are left to be transmitted, given that $i$ packets were sent during that round, with $i, j=0,1, \ldots, M$. For now, focus on a group of exactly $M$ packets, and organize the probabilities in a $(M+1) \times(M+1)$ matrix $\boldsymbol{P}_{M}=\left(p_{i j}\right)$ as in equation (9). Note that $p_{i j}=\left(\begin{array}{c}i \\ i-j\end{array}\right) p^{(i-j)}(1-p)^{j}$ for $j \leq i$, otherwise $p_{i j}=0$.

Define now $t_{i}$ as the time required to complete a round if $i$ packets are left to be transmitted. Define also $T(m)=$ $m T_{d}+2 \tau_{d_{r}}+T_{a}$ as the time needed to perform a round when the burst to send contains $m$ packets. We can organize the $t_{i} \mathrm{~s}$, $i=1, \ldots, M$ in a vector as

$$
\boldsymbol{T}_{M}=\left[\begin{array}{lllll}
0 & T(1) & \ldots & T(M-1) & T(M)
\end{array}\right] .
$$

Note that we do not need a matrix here, as the duration of the round depends only on the number of packets left to transmit, $i$. A similar argument holds for the vector $\boldsymbol{E}_{M}$, where each entry $e_{i}$ represents the energy expenditure in a round where $i$ packets are left to be transmitted. By defining $E(m)=\left(m T_{d}+\right.$ $\left.T_{a}\right) P_{T}\left(d_{r}\right)+\left(m T_{d}+T_{a}+2 \tau\right) P_{R}$, we have

$$
\boldsymbol{E}_{M}=\left[\begin{array}{lllll}
0 & E(1) & \ldots & E(M-1) & E(M)
\end{array}\right] .
$$

In general, the number of packets to be sent by a certain relay $x$ (say, $N_{x}$ ) may be greater or less than $M$, depending on the position that $x$ holds in the line. If $N_{x}>M$, the matrix $\boldsymbol{P}$ describing the transmission process can be derived by replicating $N-M$ times the first row of $\boldsymbol{P}_{M}$ as follows:

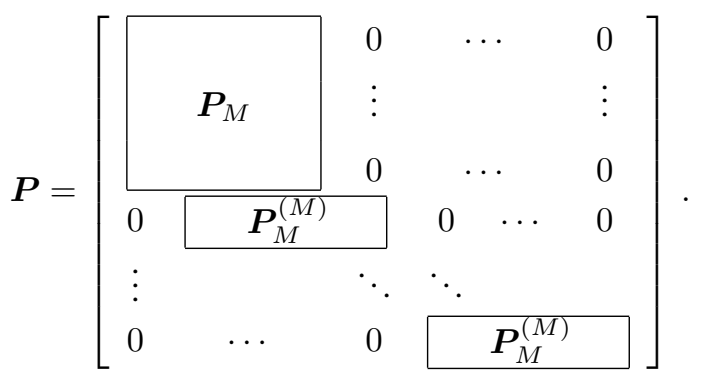

It can be noticed that the elements of $\boldsymbol{P}_{M}^{(M)}$ "shift" to the right, because no more than $M$ transmission successes are allowed with a window of length $M$, even if more than $M$ packets are left to be transmitted. In turn, this causes some states to be unreachable in only one transition. $\boldsymbol{T}$ and $\boldsymbol{E}$ are similarly constructed as follows:

$$
\begin{aligned}
& \boldsymbol{T}=\left[\begin{array}{lllll}
0 & T(1) & \ldots & T(M-1) & T(M) \ldots T(M)
\end{array}\right], \\
& \boldsymbol{E}=\left[\begin{array}{llllll}
0 & E(1) & \ldots & E(M-1) & E(M) \ldots E(M)
\end{array}\right] .
\end{aligned}
$$

In (12), the superscript ${ }^{(i)}$ denotes the $i$ th row of the corresponding matrix. If $N_{x} \leq M, \boldsymbol{P}$ is formed by extracting the submatrix containing the first $N_{x}$ rows and columns of $\boldsymbol{P}_{M}$, whereas $\boldsymbol{T}$ and $\boldsymbol{E}$ contain the first $N_{x}$ elements of $\boldsymbol{T}_{M}$ and $\boldsymbol{E}_{M}$, respectively. The overall delay and energy consumption can then be calculated using the theory of renewal reward processes, by solving a linear system of equations of the form

$$
\xi_{i 0}=\chi_{i}+\sum_{k \neq 0} p_{i k} \xi_{k 0}, \quad i=1, \ldots, N_{x},
$$

where $\chi_{i}$ is the average metric (here, delay or energy) accumulated in state $i$ before the transition. The desired variable is $\xi_{N_{x} 0}$, that represents the average metric accumulated when reaching state 0 from state $N_{x}$. To calculate the average delay $D^{(M R)}, \chi_{i}$ is set equal to $\boldsymbol{T}(i)$, i.e., the $i$ th element of $\boldsymbol{T}$. Accordingly, to calculate the average energy consumption $E^{(M R)}, \chi_{i}=\boldsymbol{E}(i)$. Note that, in this last case, the results 
must be divided by the total number of packets to be sent. It is worth highlighting that $\mathrm{S} \& \mathrm{~W}$ reduces to a special case of $M-\mathrm{S} \& \mathrm{~W}$ with $M=1$ if a feedback message is always sent after any packet reception, either correct or erroneous.

\section{Parallel transmission}

This policy is meant to exploit the larger bandwidth available on shorter links. More specifically, it works like the relaying policy, except for the fact that instead of sending all packets to the subsequent node, each relay sends in parallel a fixed number of packets $k$ directly to the destination node. We suppose that both the center frequencies and bit rates are properly chosen so that the two signals can be transmitted in non-overlapping bands. This policy will yield higher energy consumption, due to the longer transmission range, but also shorter delays, since each relay has to transmit only $N-k$ packets to the next neighbor, resulting in $(N-k) h(h+1) / 2$ total packet transmissions on the short links.

In order not to use S\&W ARQ, which is inefficient if propagation delays are long, we choose to increase the SNR to a value that ensures a very low probability $p_{t}$ that even a single packet is lost. Denoting by $\varepsilon=1-p_{t}$ the probability that all packets sent on the long link are received correctly, the required SNR $S N R_{0}^{\prime}$ must satisfy

$$
\varepsilon=\left(1-\frac{1}{2} \operatorname{erfc} \sqrt{S N R_{0}^{\prime}}\right)^{k L}
$$

which yields

$$
S N R_{0}^{\prime}=\left[\operatorname{erfc}^{-1}\left(2\left(1-\varepsilon^{1 / k L}\right)\right)\right]^{2} .
$$

Then the transmission power can be calculated as

$$
P_{T}\left(d_{i}\right)=\frac{S N R_{0}^{\prime} B\left(d_{i}\right) N_{d_{i}}}{A_{d_{i}}^{-1}},
$$

where $d_{i}$ is the distance separating the $i$ th node from the destination, $i=1, \ldots, h$. Note that as such, $P_{T}\left(d_{i}\right)$ will decrease with decreasing distance. All remaining $N-k$ packets are relayed through the nodes downstream from the source, and follow the same rules as for ordinary relaying.

The total delay for this policy depends on the number of relays between the first transmitter and the destination, as well as on the number of packets sent over the short links. Specifically, the delay is the maximum between

$$
D_{a}^{(P T)}=(N-k) \frac{h(h+1)}{2}\left(\frac{T_{d}+2 \tau_{d_{r}}}{p}+T_{a}\right)
$$

and

$$
D_{b}^{(P T)}=h k T_{d}+\tau_{d_{\ell}} .
$$

Note that $D_{b}^{(P T)}$ corresponds to the time needed by each node to transmit $k$ packets plus one propagation delay (no feedback is received for packets sent directly to the sink). We assume that each node begins its transmission exactly after overhearing the $k$ th packet from the node upstream, so that the packets sent on the long links arrive back-to-back to the sink. The energy consumption for this policy can be calculated as in (19). Also note that the last two policies listed so far, namely relaying with $M-\mathrm{S} \& \mathrm{~W}$ and parallel transmission, can be blended into a third policy that makes use of both $M-\mathrm{S} \& \mathrm{~W}$ ARQ (on short links) and long-range transmissions, in order to achieve a further delay improvement.

\section{RESUlts}

\section{A. Setting and parameters}

In the scenario considered for this analysis, the distance between the first node and the sink is $50 \mathrm{~km}$. Each node generates $N=10$ packets. Each data packet is $L=2000$ bits long, whereas ACKs are 200 bits long. The transmit power $P_{T}$ is calculated so as to guarantee a target SNR of $8 \mathrm{~dB}$ at the next relay. For long-range transmissions, the target probability of error has been set to $p_{t}=10^{-3}$ (the transmission power is set to guarantee a suitable SNR to enforce $p_{t}$ ). The channel model as outlined in Section II is fully taken into account. The sound propagation speed in water has been fixed to $1.5 \mathrm{~km} / \mathrm{s}$. For both long- and short-range transmissions, the data rate is fixed to $1 \mathrm{kbps}$. The receive power is assumed to be negligibly small with respect to the transmit power. The performance of the various techniques has been evaluated analytically in terms of the total delay and total power consumption. Both metrics have been normalized for easier comparison, the delay to the time it takes to transmit all the $h N$ packets to the sink, the energy to the amount consumed for transmitting one packet from the farthest node to the sink, assuming no errors in both cases.

\section{B. Performance Analysis}

In all pictures presented hereon, we will use the following abbreviations: RSW (Relaying with S\&W), RMSW (Relaying with $M-\mathrm{S} \& \mathrm{~W})$, PT (Parallel Transmission).

Figures 1 and 2 illustrate the results. The first and most important observation is that parallel transmissions offer a considerably shorter average data delivery time with respect to hop-by-hop relaying, and more so for greater values of $k$. The corresponding price is the increase in the average perpacket energy consumption, driven by the need to ensure a higher SNR on longer links. Noticeably, an appropriate selection of $k$ is necessary to make the energy increase worthy by improving the delay performance. From Figure 2, it is clear that the relaying with $\mathrm{S} \& \mathrm{~W}$ or $M-\mathrm{S} \& \mathrm{~W}$ yields almost the same energy consumption, and that this amount is much smaller than obtained with any parameter configuration of the parallel transmission policy. Additionally, Figure 1 shows that a more effective ARQ policy such as $M-\mathrm{S} \& \mathrm{~W}$ can outperform parallel transmissions from the point of view of delay for

$$
E^{(P T)}=(N-k) \frac{h(h+1)}{2}\left(\frac{T_{d} P_{T}\left(d_{r}\right)+\left(T_{d}+2 \tau_{d_{r}}\right) P_{R}}{p}+\left(P_{T}\left(d_{r}\right)+P_{R}\right) T_{a}\right)+\sum_{k=1}^{h} P_{T}\left(d_{k}\right) k T_{d}+\tau_{d_{\ell}}
$$




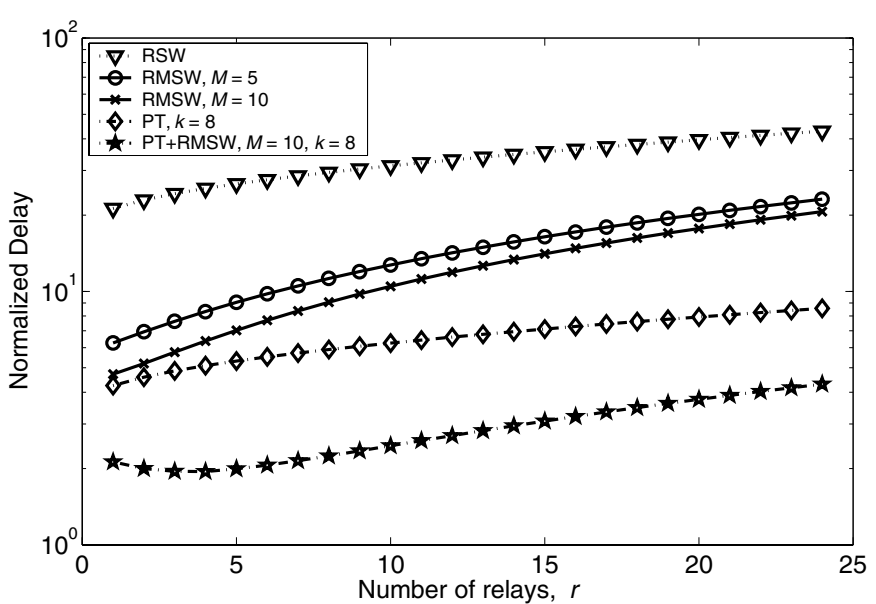

Fig. 1. Normalized delay vs. number of relays.

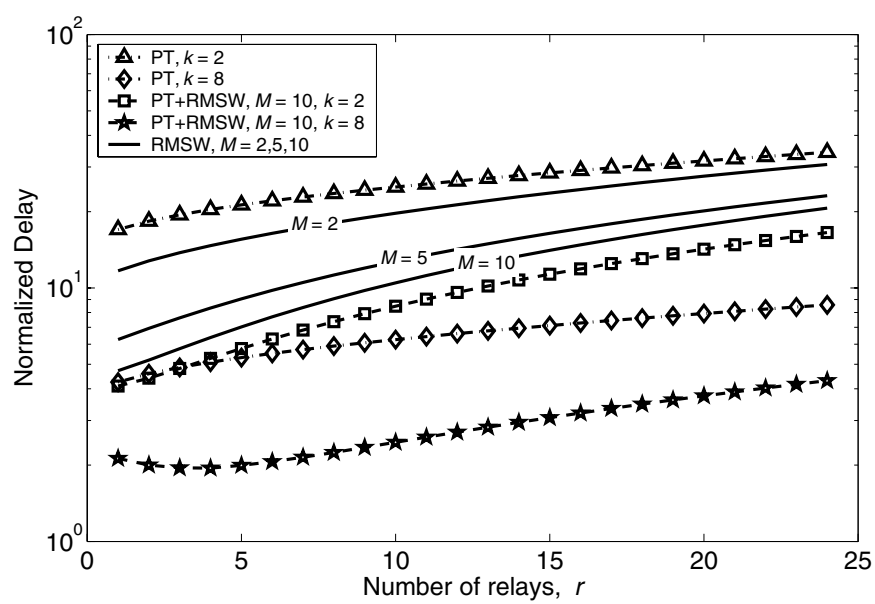

Fig. 3. Normalized delay for varying $M$ for relaying with $M-\mathrm{S} \&$ Wvs. number of relays, compared to all policies.

some values of the number of relay nodes, if $k$ is not properly chosen. This fact motivates us to blend parallel transmissions and $M-\mathrm{S} \& \mathrm{~W}$, so as to achieve the delay reduction advantages of both techniques and compensate more efficiently for the energy consumption increase.

Figures 3 and 4 add more to this argument, by showing the variation of delay and energy consumption for relaying with $M-\mathrm{S} \& \mathrm{~W}$, for different values of $M$, the length of the burst of packets after which a cumulative ACK is sent. Figure 4 suggests that relaying with $M-\mathrm{S} \& \mathrm{~W}$ yields basically the same energy consumption for any value of $M$, and that joining parallel transmissions and relaying with $M-\mathrm{S} \& \mathrm{~W}$ helps keep the expenditure lower even for the more energydemanding parallel transmission policies. Delay is impacted more significantly by the choice of parameters. For example, the parallel transmission policy with $k=2$ is outperformed by all relaying with $M-\mathrm{S} \& \mathrm{~W}$ policies, for any chosen value of $M .^{2} \quad$ If $k=8$, parallel transmissions yield better performance than relaying with $M-\mathrm{S} \& \mathrm{~W}$ but at the price of a 5- to 7fold increase in energy. On the contrary, using an effective relaying policy with $M-\mathrm{S} \& \mathrm{~W}(e . g .$, with $M=10)$ along with

\footnotetext{
${ }^{2}$ Note that increasing $M$ yields progressively smaller incremental advantages, which is in agreement with the results in [13].
}

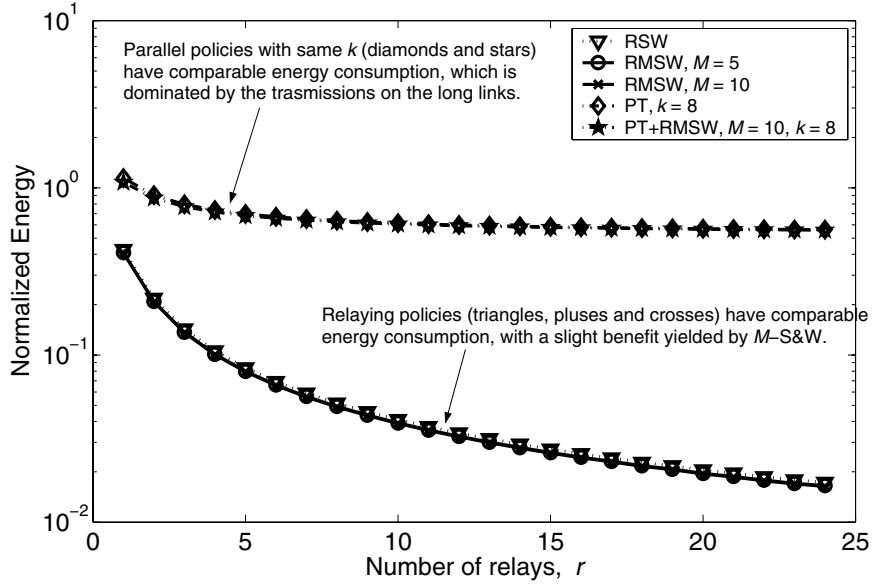

Fig. 2. Normalized energy per packet vs. number of relays.

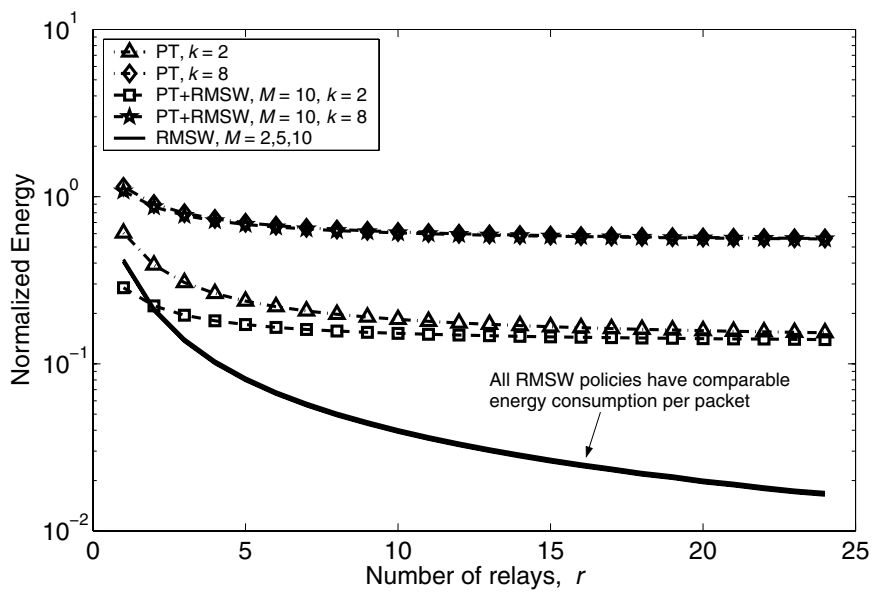

Fig. 4. Normalized energy per packet for varying $M$ in RMSW vs. number of relays, compared to all policies.

parallel transmissions with a low $k=2$, improves considerably the parallel transmission delay performance, and may become even better than relaying with $M-\mathrm{S} \& \mathrm{~W}$ from the point of view of energy consumption, if a low number of relays is present. A different design strategy with $k=8$ and $M=10$, instead, would give even further delay advantages, at the price of the same energy increase.

To provide more insight into the effects of $k$ on the performance of parallel transmissions with and without $M-\mathrm{S} \& \mathrm{~W}$, Figures 5 and 6 show the delay and energy metrics for varying $k$ in the parallel transmission policy. It is clear from Figure 5 that a suboptimal choice of $k$ does not decrease the average delay sufficiently to compensate for the energy increase. For example, while $k=2$ is outperformed by any other relaying with $M-\mathrm{S} \& \mathrm{~W}$ policy, for $k=6$ the performance improvement begins to become noticeable, whereas $k=8$ gives better performance than all relaying with $M-\mathrm{S} \& \mathrm{~W}$ policies investigated. Again, parallel transmissions with $M-\mathrm{S} \& \mathrm{~W}$ ARQ on short links yields the best delay results if $k$ is sufficiently high, though being more energy-demanding.

Figure 7 finally summarizes the observations reported so far, by comparing energy against delay for a number of different policies. Each curve is spanned counterclockwise from its top-left point by increasing the number of relays. 


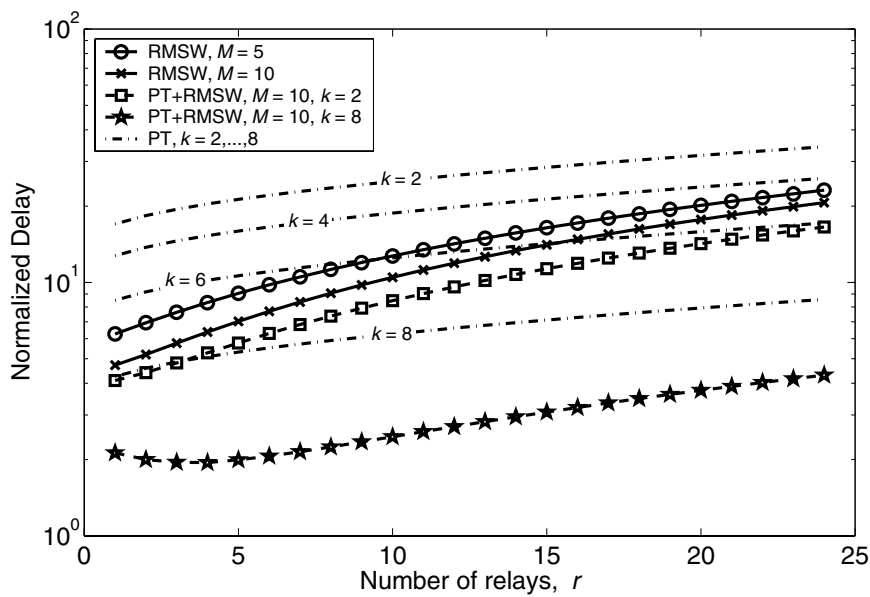

Fig. 5. Normalized delay for varying $k$ in PT vs. number of relays, compared to all policies.

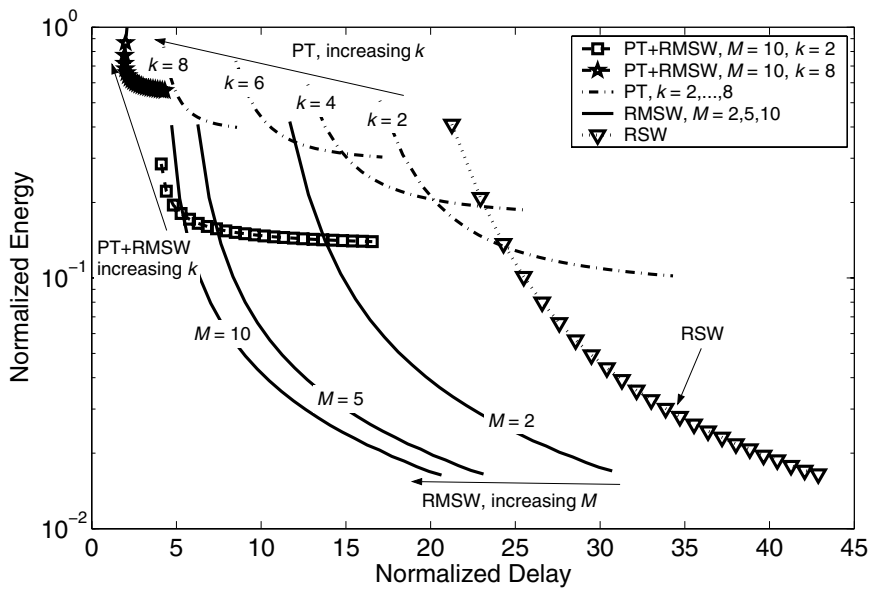

Fig. 7. Energy per packet vs. delay comparison for all policies vs. number of relays.

The most "favorable corner" in this picture is the bottom-left one, where both energy and delay are low. We notice that any parallel transmissions policy immediately increases the energy consumption and yields significant delay improvements only for high $k$, motivating the integration of parallel transmissions and relaying with $M-\mathrm{S} \& \mathrm{~W}$. Moreover, increasing $k$ limits the variation of the curves with respect to the energy axis, as longrange transmissions become the most important contributors to the energy consumption. In summary, relaying with $\mathrm{S} \& \mathrm{~W}$ or $M-\mathrm{S} \& \mathrm{~W}$ yields the most favorable energy performance, whereas using parallel transmissions (with or without relaying using $M-\mathrm{S} \& \mathrm{~W}$ on the short links) can provide shorter delay at the price of a possibly substantial energy increase.

\section{CONCLusions}

In this paper, we derived analytically the delivery delay and energy consumption performance of different data forwarding policies, considering a simple, yet insightful line topology in which the packets must be delivered to an end sink node. We suggested the use of parallel transmissions over both a shortrange, high-frequency link and a long-range, low-frequency one, and showed how policy parameters affect the metrics of

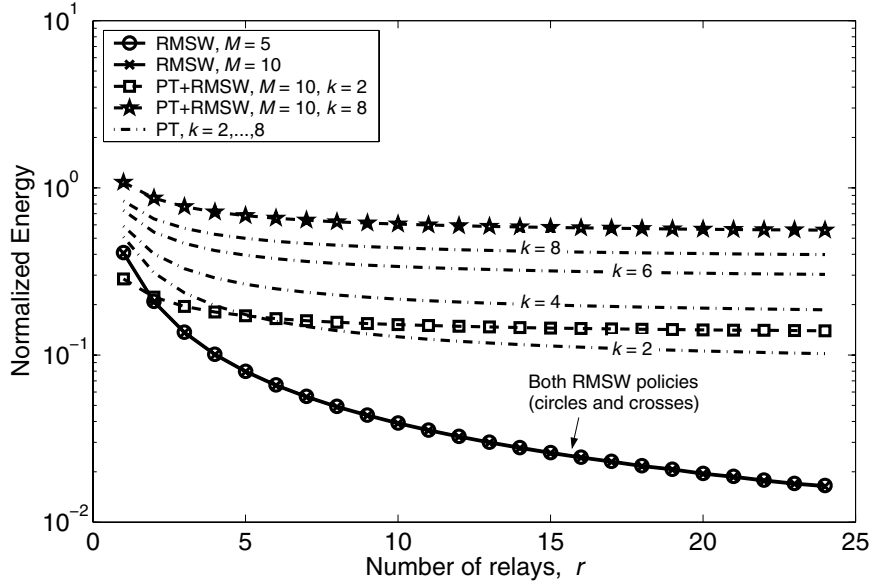

Fig. 6. Normalized energy per packet for varying $k$ in PT vs. number of relays, compared to all policies.

interest. We also pointed out that the different policies offer a way to trade off delay for energy consumption in underwater networks. Future work on this topic will include modeling the channel fading and the effect of packet coding techniques on delay and energy performance.

\section{REFERENCES}

[1] M. Stojanovic, "On the relationship between capacity and distance in an underwater acoustic communication channel," in Proc. ACM WUWNet, Los Angeles, CA, Sept. 2006, pp. 41-47.

[2] E. M. Sozer, M. Stojanovic, and J. G. Proakis, "Underwater acoustic networks," IEEE/MTS J. Oceanic Eng., vol. 25, no. 1, pp. 72-83, Jan. 2000.

[3] P. Casari, S. Marella, and M. Zorzi, "A comparison of multiple access techniques in clustered underwater acoustic networks," in Proc. IEEE/OES Oceans, Aberdeen, Scotland, June 2007.

[4] B. Peleato and M. Stojanovic, "A MAC protocol for ad hoc underwater acoustic sensor networks," in Proc. ACM WUWNet, Los Angeles, CA, Sept. 2006, pp. 113-115.

[5] A. Syed, W. Ye, and J. Heidemann, "Medium access control for underwater acoustic networks," in Proc. ACM WUWNet, Los Angeles, CA, Sept. 2006, work-in-progress paper.

[6] M. K. Park and V. Rodoplu, "UWAN-MAC: an energy-efficient MAC protocol for underwater acoustic wireless networks," IEEE/MTS J. Oceanic Eng., 2007, to appear. [Online]. Available: http://www.ece. ucsb.edu/rodoplu/Pubs/ParkRodoplu UWANMAC.pdf

[7] P. Xie, J.-H. Cui, and L. Lao, "VBF: vector-based forwarding protocol for underwater sensor networks," UCONN CSE, Tech. Rep. UbiNetTR05-03 (BECAT/CSE-TR-05-6), Feb. 2005.

[8] P. Xie and J.-H. Cui, "SDRT: a reliable data transport protocol for underwater sensor networks," UCONN CSE, Tech. Rep. UbiNet-TR0603, Feb. 2006

[9] D. Pompili, T. Melodia, and I. F. Akyildiz, "Routing algorithms for delay-insensitive and delay-sensitive applications in underwater sensor networks," in Proc. ACM Mobicom, 2006.

[10] A. F. Harris III and M. Zorzi, "Energy-efficient routing protocol design considerations for underwater networks," in Proc. IEEE SECON, June 2007.

[11] P. Casari and A. F. Harris III, "Energy-efficient reliable broadcast in underwater acoustic networks," in Proc. of ACM WUWNet, Montréal, Canada, Sept. 2007.

[12] L. Berkhovskikh and Y. Lysanov, Fundamentals of Ocean Acoustics. Springer, 1982.

[13] M. Stojanovic, "Optimization of a data link protocol for an underwater acoustic channel," in Proc. IEEE Oceans, Brest, France, June 2005, pp. $68-73$.

[14] L. Rizzo and L. Vicisano, "RMDP: An FEC-based Reliable Multicast Protocol for Wireless Environments," ACM SIGMOBILE Mobile Computing and Communications Review, vol. 2, no. 2, pp. 23-31, Apr. 1998. 